\title{
Pharmacodynamic change in plasma angiogenic proteins: a dose-escalation phase 1 study of the multi-kinase inhibitor lenvatinib
}

\author{
Noriyuki Koyama', Kenichi Saito², Yuki Nishioka ${ }^{3}$, Wataru Yusa ${ }^{4}$, Noboru Yamamoto 5 , Yasuhide Yamada \\ Hiroshi Nokihara ${ }^{5}$, Fumiaki Koizumi ${ }^{7}$, Kazuto Nishio ${ }^{8}$ and Tomohide Tamura ${ }^{5^{*}}$
}

\begin{abstract}
Background: Lenvatinib (E7080), an oral multi-kinase inhibitor, has inhibitory action on tumor cell proliferation and tumor angiogenesis in preclinical models. We evaluated correlations between pharmacodynamic (PD) biomarkers with patient clinical outcomes in a lenvatinib phase 1 dose-escalation study.

Methods: Plasma angiogenic proteins were evaluated as potential PD biomarkers of response to lenvatinib in a dose-escalation phase 1 study. Lenvatinib was administered to 27 patients by twice-daily dosing in 3-week cycles; 2 weeks of treatment followed by 1 week of rest until discontinuation. Blood samples for plasma proteins were collected on days 1 (baseline), 8, and 15 of cycle 1, and days 1, 8, and 15 of cycle 2. Selected clinical outcomes, including tumor shrinkage and adverse events (AEs), were used for correlative analyses of pharmacokinetic parameters and PD biomarkers.

Results: Tumor shrinkage and changes in PD biomarkers (increased vascular endothelial growth factor [VEGF] and stromal cell-derived factor 1 alpha [SDF1a] levels and decreased soluble VEGF receptor 2 [SVEGFR2] levels) significantly correlated with increasing lenvatinib exposure. Observed changes in levels of VEGF, SDF1a, and SVEGFR2 were maintained on day 15 of cycle 1, but returned to baseline during the 1-week rest period, and similar changes were induced by reinstitution of treatment in cycle 2 . The worst grades of hypertension, proteinuria, and fatigue were associated with changes in VEGF and HGF at day 8 of cycle 1. Maximum tumor shrinkage was correlated with increased SDF1a levels. Decreased sVEGFR2 level was also correlated with tumor shrinkage and frequency of hypertension, proteinuria, and fatigue. Tumor shrinkage significantly correlated with the worst grade of proteinuria, but not with hypertension or fatigue.
\end{abstract}

Conclusion: PD biomarker changes observed in plasma angiogenic proteins are correlated with lenvatinib-induced tumor shrinkage and AEs. Our findings warrant further assessment of plasma proteins associated with angiogenesis as potential biomarkers of lenvatinib activity.

Trial registration: ClinicalTrial.gov: NCT00280397 (January 20, 2006).

Keywords: Lenvatinib, Angiogenesis, Pharmacodynamic biomarkers, VEGF, SDF1a, sVEGFR2, Maximum tumor shrinkage

\section{Background}

Various agents that inhibit tumor angiogenesis have recently been approved or are currently being developed in clinical trials [1-4]. Although treatment benefits are often seen early during the course of antiangiogenic therapy, therapy is often discontinued when tumors develop resistance and resume

\footnotetext{
* Correspondence: ttamura@ncc.go.jp

${ }^{5}$ Department of Thoracic Oncology, National Cancer Center Hospital, Tokyo, Japan

Full list of author information is available at the end of the article
}

growth. Additionally, accumulation of biologic changes in host tissue may result in unacceptable toxicities that necessitate dose interruptions or reductions, resulting in decreased dose density and potentially lower efficacy.

Compensatory mechanisms for resistance may be acquired by the tumor and host tissues as a response to vascular damage and elevated tumor hypoxia, and include upregulation of alternative proangiogenic factors. A recent study indicated that stable microvasculature kept disseminated tumor cells dormant, whereas sprouting neovasculature sparked 
micrometastatic outgrowth [5]. Proangiogenic factors derived from tumor tissues include platelet-derived growth factor (PDGF), placental growth factor (PlGF), basic fibroblast growth factor (bFGF), and stromal cell-derived factor1 alpha $(\mathrm{SDF} 1 \alpha)$. Stromal cells surrounding a tumor, such as tumor-associated fibroblasts, can upregulate PDGF-C and activate pericytes, which also play a role in maintaining vascular integrity and developing resistance in response to inhibition of vascular endothelial growth factor (VEGF) [6]. In addition, a variety of bone-marrow-derived cells may mediate resistance to VEGF inhibition by producing proangiogenic factors $[7,8]$. Some tumors develop resistance to VEGF inhibitors by secreting cytokines that recruit myeloid cells and other cells that promote angiogenesis and immune tolerance, thereby affecting the efficacy and safety of anti-VEGF therapy [9].

The development of biomarkers of clinical efficacy and safety may provide important clinical insight for the appropriate selection of patients and management of antiangiogenesis therapy. Early prediction of efficacy and toxicity with plasma biomarkers related to angiogenesis may contribute to optimal patient care. In addition, potential insight into the mechanisms of resistance may lead to the development of rational combinations of antiangiogenic treatment with agents that inhibit other signaling pathways that promote resistance to antiangiogenic therapy $[1,10]$.

Over the past decade, a multiplex protein assay has been validated that enables identification of multiple changes in the levels of plasma proteins in preclinical and clinical samples. In preclinical studies, treatment with the VEGF receptor (VEGFR) inhibitor sunitinib induced dose-dependent increases in VEGF and PIGF levels and decreases in soluble VEGFR 2 (sVEGFR2) levels, while treatment with cetuximab, an epidermal growth factor receptor antibody, increased transforming growth factor alpha levels in a tumor-independent manner [11,12]. These data suggest that changes in the levels of plasma proteins may reflect the biologic response of host tissues to therapy and may be useful markers for the clinical activity of antitumor agents.

Lenvatinib (E7080) is an oral multiple tyrosine kinase inhibitor (TKI) of VEGFR1-3, fibroblast growth factor receptor $1-4$, PDGF receptor alpha $(-\alpha)$, RET protein, and $\mathrm{c}$-Kit protein. Inhibition of xenograft tumor growth by lenvatinib was observed at doses as low as 1.0 and $10.0 \mathrm{mg} / \mathrm{kg}$ [13-15]. In phase 1 and 2 clinical trials, lenvatinib demonstrated antitumor activity and a manageable toxicity profile as a single agent [16-18]. In a phase 1 dose-escalation study, lenvatinib showed preliminary activity for durable disease control in a variety of tumor types, including a partial response in a patient with colon cancer and stable disease in $84 \%$ of evaluable patients [17]. Lenvatinib has a manageable toxicity profile with adverse events (AEs) consistent with other antiVEGF treatments, including hypertension, proteinuria, and fatigue $[16,17,19]$. In this phase 1 dose-escalation study, we analyzed the pharmacodynamic (PD) changes in angiogenic plasma proteins during cycles 1 and 2 of lenvatinib treatment.

\section{Methods}

\section{Study design}

This single-center, open-label, sequential dose-escalation study of lenvatinib was conducted at the National Cancer Center Hospital, Tokyo, Japan. Lenvatinib was orally administered twice daily in 3-week cycles (2 weeks on/1 week off) in patients with advanced solid tumors. Pharmacokinetic (PK) parameters, safety, tolerability, efficacy, and exploratory PD markers were examined. Eligible patients were sequentially enrolled on escalating doses of oral lenvatinib with a standard $3+3$ design. AEs were monitored throughout the treatment cycles. Best tumor response and disease progression were measured using the Response Evaluation Criteria in Solid Tumors (RECIST), version 1.0 [20]. Tumors were assessed at screening, in cycle 2 or 3 , and in every 2 cycles thereafter. This study was performed in accordance with the ethical principles stipulated by the Declaration of Helsinki and Good Clinical Practice guidelines, and approved by the Institutional Review Board at the National Cancer Center Hospital, Tokyo, Japan. All patients provided written, informed consent before screening.

\section{Pharmacokinetic and pharmacodynamic analyses}

Blood samples for PK and PD analyses were collected from each patient. Plasma lenvatinib concentrations were determined with liquid chromatography/tandem mass spectrometry by Sumitomo Chemical Co. Ltd (Osaka, Japan). Area under the curve (AUC) was calculated from the data obtained at steady state in cycle 1. Plasma proteins were measured with a BioPlex assay (Bio-Rad Laboratories, Inc) by Mitsubishi Chemical Medience Corp (Ibaraki, Japan). Plasma PD biomarkers measured in this study included: interleukin (IL)-6, IL-8, and IL-10; VEGF; PDGF; hepatocyte growth factor (HGF); stem cell factor (SCF); and SDF1 $\alpha$. sVEGFR1 and sVEGFR2 were measured by enzyme-linked immunosorbent assay.

\section{Statistical analysis}

PK parameters of plasma lenvatinib concentration-vs-time data were examined by noncompartmental analysis using WinNonlin version 5.2 software (Pharsight Corporation, Mountain View, CA, USA). Correlation analyses between PK, PD, and clinical outcomes were performed using Spearman's rank correlation coefficient, and Wilcoxon signed rank test was used to determine change from pretreatment. 
Multiplicity adjustments were not conducted. Maximum tumor shrinkage (\%) was defined as the percentage of change from baseline in the sum of tumor diameters of target lesions at the maximum shrinkage observed.

\section{Results}

Twenty-seven patients were enrolled in the study. Because change in plasma proteins is hypothesized to reflect biologic response to treatment and may be a marker of clinical activity, we examined whether lenvatinib treatment altered the levels of putative PD biomarkers (Figure 1). We measured a total of 20 plasma angiogenic proteins and cytokines at baseline and after treatment [17], and found that levels of IL-6, IL-10, VEGF, HGF, and SDF1 $\alpha$ were increased, whereas levels of PDGF-BB, sVEGFR1, and sVEGFR2 were decreased at day 8 of lenvatinib treatment. IL- 8 and SCF levels were increased in some patients but decreased in others.

We next investigated AUC-dependent changes in PD biomarker levels in plasma proteins and correlations with area under the curve for the dosing interval $\left(\mathrm{AUC}_{0 \text {-tau; }}\right.$ Table 1). Only the increased levels of VEGF and SDF1 $\alpha$ and the decreased level of sVEGFR2 were significantly correlated with $\mathrm{AUC}_{0 \text {-tau }}$. Correlation coefficients and $P$ values, respectively, were 0.496 and .030 for VEGF, 0.806 and $<.0001$ for SDF1 $\alpha$, and -0.916 and $<.0001$ for sVEGFR2. Similar correlations were seen in the analysis with maximum and minimum concentrations (data not shown). Relative to the dosing schedule, PD changes in these proteins were induced on day 8 of cycle 1 and maintained on day 15 of cycle 1, but returned to baseline during the 1-week rest period. Similar changes were induced by reinstitution of treatment in cycle 2 , suggesting that these PD biomarker changes were associated with lenvatinib treatment (Figure 2).

Correlation analyses of AEs and tumor shrinkage with $\mathrm{AUC}_{0 \text {-tau }}$ were also performed. In a previous study, the most frequent AEs associated with lenvatinib treatment

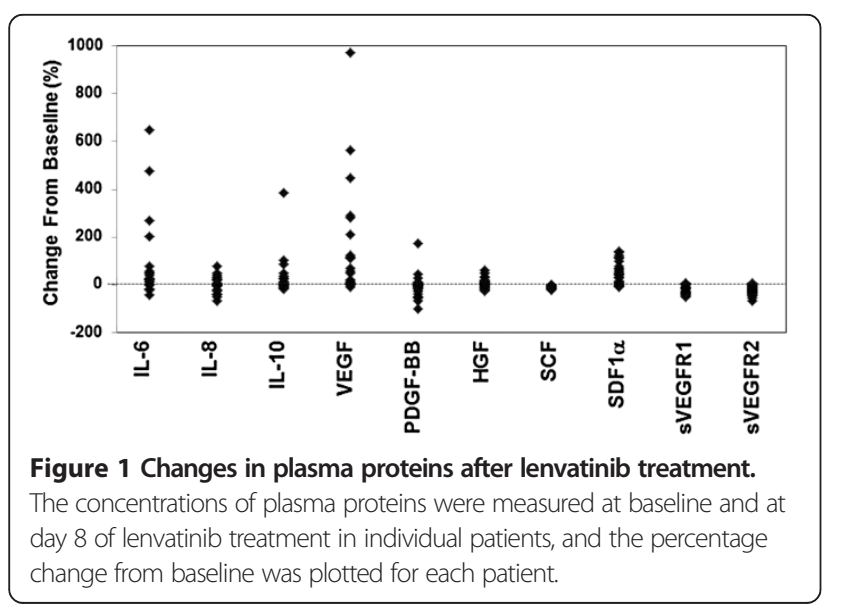

were hypertension, proteinuria, and fatigue [17]. Using the worst grade of each of these AEs over the duration of treatment in correlation with $\mathrm{AUC}_{0-\text { tau }}$, Spearman's rank correlation analysis indicated significant correlation of hypertension $(P=.005)$, proteinuria $(P=.003)$, and fatigue $(P=.017)$ with $\mathrm{AUC}_{0 \text {-tau }}$ (Figure $\left.3 \mathrm{~A}-\mathrm{C}\right)$. Correlation analyses of other AEs were not performed, because other AEs occurred in a limited number of patients [17]. The analysis of maximum tumor shrinkage and $\mathrm{AUC}_{0 \text {-tau }}$ yielded a significant but weak correlation $(P=.038$; Figure $3 \mathrm{D})$. The results of correlation analysis of toxicities and tumor shrinkage with the PD change in plasma proteins at cycle 1 are listed in Table 2 . The analysis showed a significant correlation between change in VEGF and HGF levels in cycle 1 with the worst grades of hypertension, proteinuria, and fatigue. Additionally, maximum tumor shrinkage showed a significant correlation with PD change in SDF1 $\alpha$ levels, where patients with a greater increase in SDF1 $\alpha$ levels had greater tumor shrinkage. However, no correlations with tumor shrinkage were seen for VEGF or HGF. Decreased sVEGFR2 level was also correlated with tumor shrinkage and frequency of hypertension, proteinuria, and fatigue.

Finally, a correlation analysis of AEs with maximum tumor shrinkage is shown in Figure 4. Although tumor shrinkage and worst grade in hypertension, proteinuria, and fatigue were significantly correlated with $\mathrm{AUC}_{0 \text {-tau }}$ (Figure 3), a significant correlation between tumor shrinkage and worst grade of AE was only observed for proteinuria $(P=.014$; Figure $4 \mathrm{~B})$.

\section{Discussion}

In this study, we have observed significant correlations of toxicity and tumor shrinkage with PK parameters and

Table 1 Correlation between lenvatinib treatmentdependent changes in plasma biomarkers and AUC

\begin{tabular}{llcc}
\hline $\begin{array}{l}\text { Plasma } \\
\text { Biomarker }\end{array}$ & $\mathbf{n}$ & \multicolumn{2}{c}{ Correlation With $\mathbf{A U C}_{\mathbf{0} \text {-tau }}$} \\
\cline { 3 - 4 } & & $\boldsymbol{r}$ & $\boldsymbol{P}$ Value \\
\hline IL-6 & 19 & -0.100 & .683 \\
IL-8 & 19 & -0.202 & .407 \\
IL-10 & 19 & 0.061 & .802 \\
VEGF & 19 & 0.496 & .030 \\
PDGF-BB & 19 & -0.161 & .509 \\
HGF & 25 & 0.263 & .203 \\
SCF & 25 & -0.210 & .313 \\
SDF1a & 25 & 0.806 & $<.0001$ \\
SVEGFR1 & 25 & -0.378 & .062 \\
SVEGFR2 & 25 & -0.916 & $<.0001$ \\
\hline
\end{tabular}

The concentrations of plasma proteins were measured at baseline and at day 8 of lenvatinib treatment, and the percentage change from baseline was

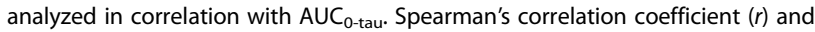
$P$ value $(p)$ for each analysis is listed. 

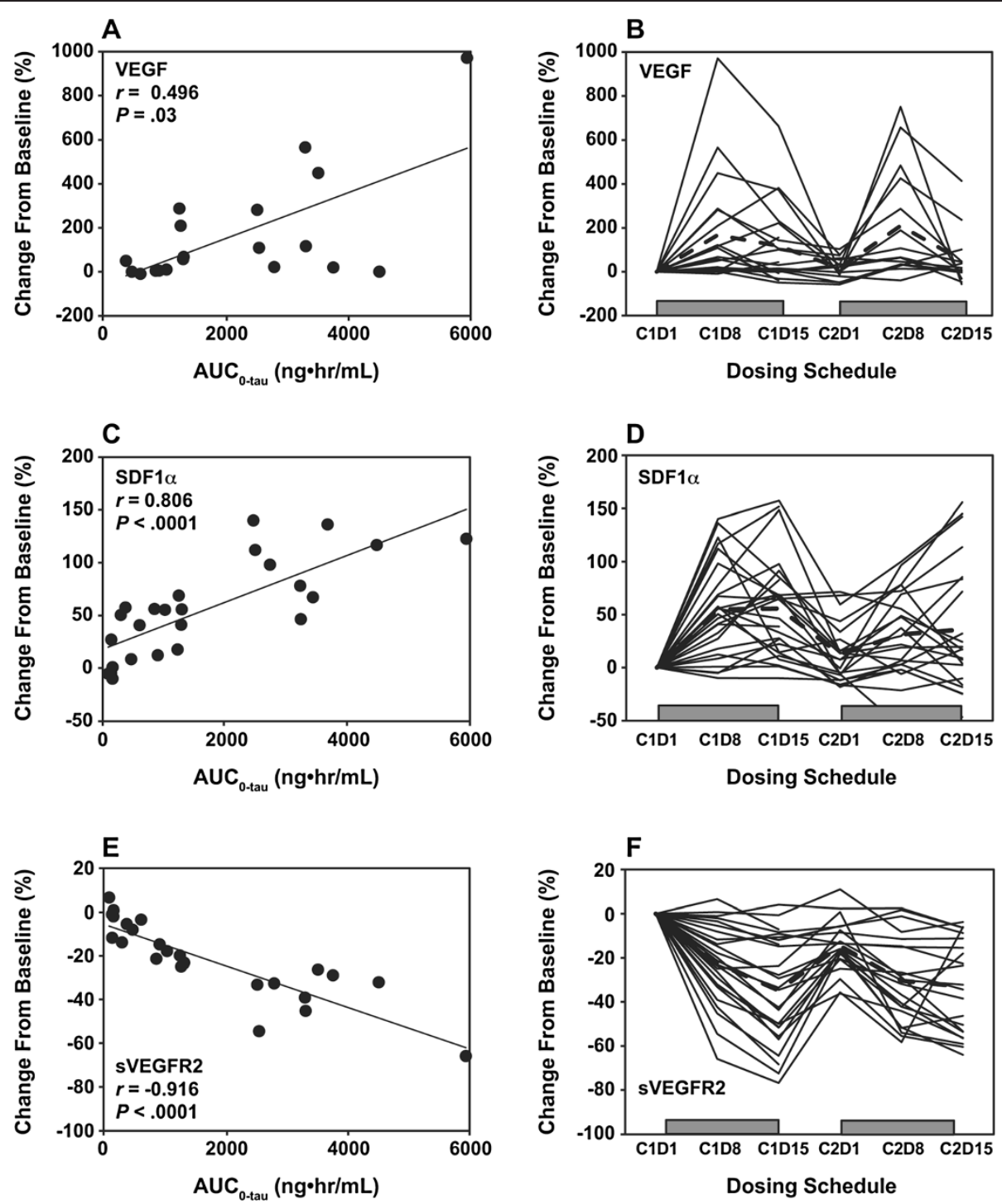

Figure 2 Lenvatinib treatment-dependent changes in VEGF, SDF1a, and sVEGFR2. The concentrations of plasma VEGF (A), SDF1a (C), and SVEGFR2 (E) were measured at baseline and at day 8 of lenvatinib treatment, and the percentage change from baseline was plotted in correlation with $\mathrm{AUC}_{0 \text {-tau }}$. The correlation coefficient $(r)$ and $P$ value in each analysis are indicated. The percentage PD changes in VEGF (B), SDF1a (D), and sVEGFR2 (F) relative to dosing schedule were indicated for 14 days on treatment (at days [D] 8 and 15 of cycle [C] 1), after 7 days off treatment, and on retreatment in cycle 2. A dotted line indicates the mean percentage of change, and gray boxes indicate each on-treatment period.

PD changes in VEGF, SDF1 $\alpha$, and sVEGFR2 levels. While evaluating $\mathrm{PK}$ parameters requires multiple samplings and analyses, PD changes in plasma markers are more easily monitored. More importantly, PD biomarkers may reflect biologic changes in tumor and host tissues in response to treatment and are potentially useful for patient monitoring.

An adaptive treatment approach based on the incidence of toxicity may be effective in maintaining treatment and increasing treatment benefits of VEGF inhibitors [19]. The development of both treatment-related hypertension and proteinuria has been reported in patients receiving lenvatinib therapy $[17,19]$, as well as in clinical studies of other inhibitors of the VEGF signaling pathway $[21,22]$. We have observed that changes in the levels of VEGF and HGF in cycle 1 correlated with the worst grade of hypertension, proteinuria, and fatigue. Monitoring plasma levels of VEGF and HGF may help predict toxicity, and by identifying those patients who require increased surveillance, it may lessen the risk of AE incidence or worsening severity.

The effects of VEGF and HGF on blood pressure may be explained by their induction of endothelial proliferation and contribution to the protection and repair of 

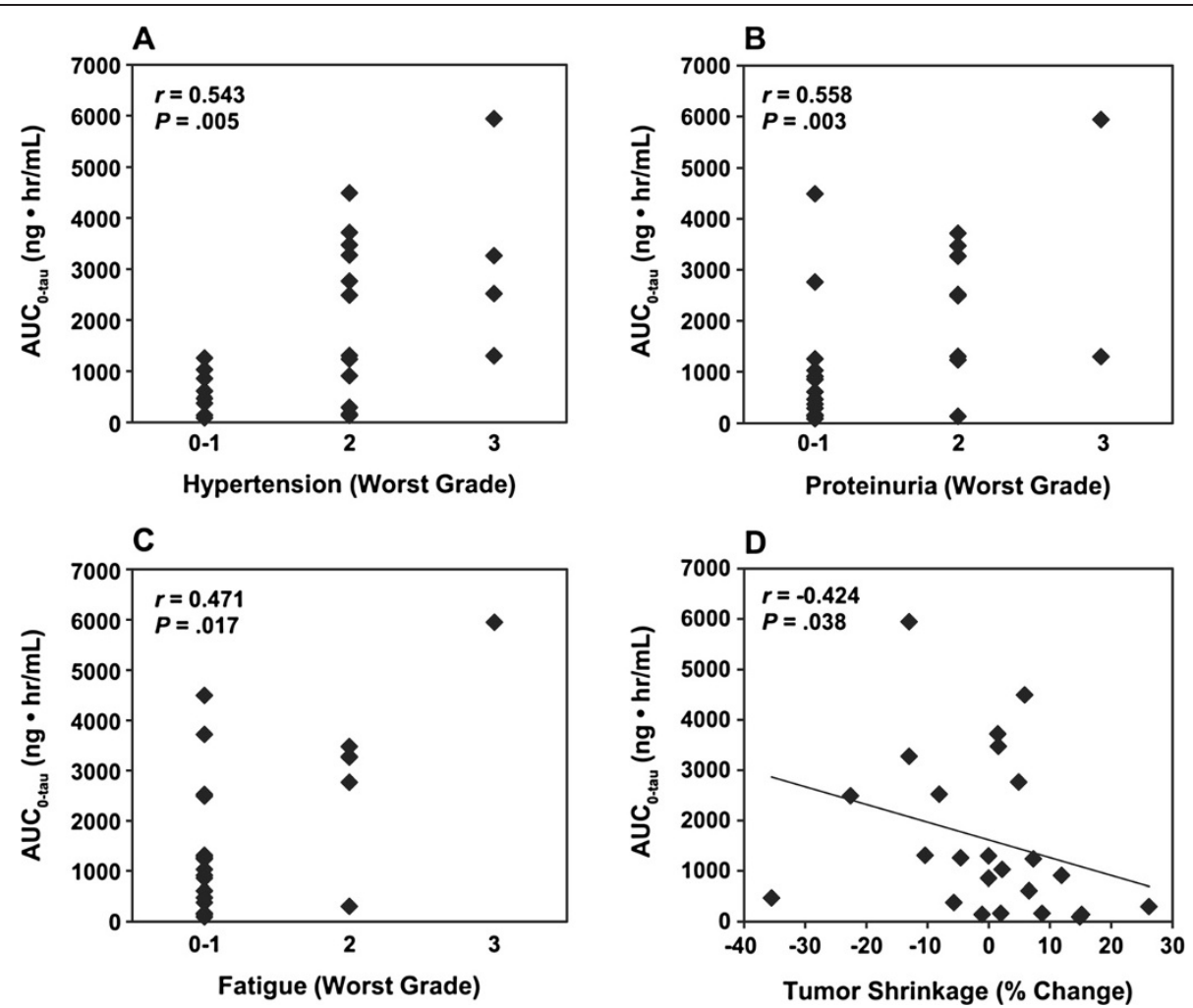

Figure 3 Spearman's correlation analysis of AUC with toxicity and tumor shrinkage induced by lenvatinib. The worst grade of hypertension (A), proteinuria (B), and fatigue (C) and the maximum tumor shrinkage (D) for the treatment duration were analyzed in correlation with $\mathrm{AUC}_{0 \text {-tau. }}$. The correlation coefficient $(r)$ and $P$ value for each analysis is indicated.

vascular endothelial cells [23]. HGF may be upregulated in response to elevated blood pressure to counter endothelial dysfunction. This concept is supported by recent reports that HGF treatment produced therapeutic benefit against peripheral arterial disease $[24,25]$.
The relationship between increased levels of VEGF and HGF with fatigue, however, is not clear. Elevated VEGF was significantly associated with increased fatigue in anthracycline-based chemotherapy in breast cancer [26]. Additionally, correlations were reported between

Table 2 Correlation of toxicities and tumor shrinkage with percentage change in plasma biomarkers

\begin{tabular}{|c|c|c|c|c|c|c|c|c|c|}
\hline \multirow{2}{*}{$\begin{array}{l}\text { Plasma } \\
\text { Biomarker }\end{array}$} & \multirow[t]{2}{*}{$\mathrm{n}$} & \multicolumn{2}{|c|}{ Hypertension } & \multicolumn{2}{|c|}{ Proteinuria } & \multicolumn{2}{|c|}{ Fatigue } & \multicolumn{2}{|c|}{ Tumor Shrinkage } \\
\hline & & $r$ & $P$ Value & $r$ & $P$ Value & $r$ & $P$ Value & $r$ & $P$ Value \\
\hline$\overline{I L-6}$ & 19 & 0.19 & .421 & 0.247 & .294 & -0.173 & .465 & -0.437 & .061 \\
\hline IL-8 & 19 & -0.077 & .748 & 0.053 & .823 & 0.002 & .993 & -0.191 & .434 \\
\hline IL-10 & 19 & 0.158 & .505 & 0.038 & .872 & 0.141 & .552 & -0.392 & .097 \\
\hline VEGF & 19 & 0.569 & $.008^{\mathrm{b}}$ & 0.703 & $<.001^{\mathrm{a}}$ & 0.529 & $.016^{c}$ & -0.277 & .250 \\
\hline PDGF-BB & 19 & -0.215 & .363 & -0.151 & .524 & 0.043 & .857 & -0.277 & .250 \\
\hline HGF & 25 & 0.624 & $<.001^{a}$ & 0.615 & $<.001^{a}$ & 0.431 & $.027^{c}$ & -0.235 & .257 \\
\hline SCF & 25 & 0.145 & .478 & 0.176 & .390 & -0.057 & .780 & 0.261 & .207 \\
\hline SDF1a & 25 & 0.257 & .204 & 0.314 & .117 & 0.344 & .085 & 0.424 & $.034^{c}$ \\
\hline sVEGFR1 & 25 & -0.38 & .055 & -0.365 & .066 & 0.065 & .753 & 0.038 & .855 \\
\hline sVEGFR2 & 25 & -0.613 & $<.001^{\mathrm{a}}$ & -0.601 & $.001^{\mathrm{b}}$ & -0.466 & $.016^{c}$ & -0.431 & $.031^{c}$ \\
\hline
\end{tabular}

The concentrations of plasma proteins were measured at baseline and at day 8 of lenvatinib treatment, and the percentage change from baseline was analyzed (Spearman's correlation analysis) in correlation with toxicities and tumor shrinkage. Worst grade of toxicities and maximum tumor shrinkage over the duration of treatment were used for the analysis.

${ }^{\mathrm{a}} P<0.001$.

${ }^{\mathrm{b}} P<0.01$.

${ }^{c} P<0.05$. 

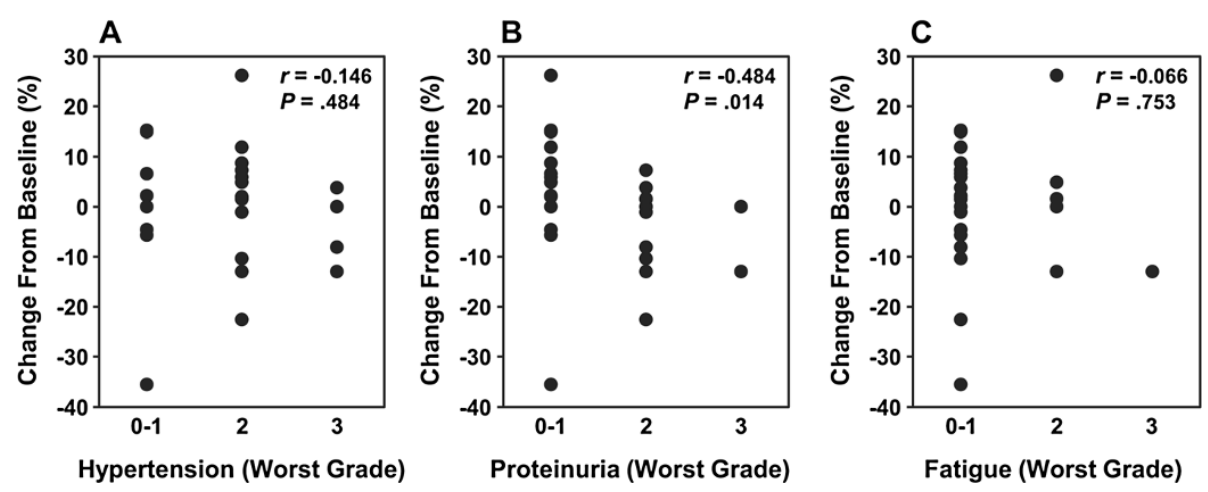

Figure 4 Correlation of tumor shrinkage with the worst grade of toxicity. Correlation analyses were performed for maximum tumor shrinkage percentage change from baseline and the worst grade of hypertension $(\mathbf{A})$, proteinuria $(\mathbf{B})$, and fatigue $(\mathbf{C})$ over the treatment duration. The correlation coefficient $(r)$ and $P$ value for each analysis is indicated.

lower serum HGF levels and fatigue in healthy control participants, as well as between increased serum HGF levels and antidepressant efficacy in patients with panic disorder [27]. Hypothyroidism has been reported in sorafenibtreated patients with renal cancer and sunitinib-treated patients with gastrointestinal stromal cancer [28,29], and anti-VEGF or anti-VEGFR2 treatment induced vascular regression in the thyroid and decreased plasma thyroid hormone levels in mice [30]. Additionally, thyroid hormone replacement therapy improved fatigue in axitinib-treated patients with cancer [31]. These reports support the need for further biomarker analyses to elucidate the role of VEGF and HGF in thyroid function.

Increased SDF1 $\alpha$ levels were also correlated with greater tumor shrinkage. Activation of the immune pathway is important for tumor shrinkage; SDF1 $\alpha$ and its receptor CXCR4 play important roles in immune function and have the potential to enhance anticancer immunity [32,33]. SDF1 $\alpha$ and CXCR4 also enhance progenitor cell accumulation at angiogenic sites and are important biomarkers of antiangiogenic therapy resistance [34]. We have previously reported that higher baseline SDF1 $\alpha$ levels correlated with shorter treatment duration [17]. The role of SDF1 $\alpha$ as a potential PD biomarker of resistance to lenvatinib treatment needs further study, especially because baseline and subsequent changes from baseline levels of SDF1 $\alpha$ may be interpreted differently.

VEGFR2 is one of the most important mediators of angiogenesis in normal and tumor tissues [35]. We have observed decreases in levels of sVEGFR1 and sVEGFR2 after lenvatinib treatment, while decreased sVEGFR2 levels were correlated with $\mathrm{PK}$ parameters, AE frequency, and tumor shrinkage. Soluble forms of VEGFR1 and VEGFR2 are induced through alternative splicing of VEGFR1 and VEGFR2 transcripts and act as inhibitors of VEGF signaling [36,37]. TKI treatment-associated decreases in circulating sVEGFR2 levels have been consistently observed [38-40], but their clinical relevance remains controversial.
A possible interpretation is that a decreased level of sVEGFR2 is a surrogate index for PK parameters such as AUC, which was correlated with both AEs and tumor shrinkage in our phase 1 study. A study of axitinib in renal cell carcinoma indicated that patients with greater decreases in sVEGFR2 levels showed higher objective response rates and longer progression-free survival (PFS) than those with smaller decreases [41]. Recent results of a trial evaluating cediranib in hepatocellular carcinoma found that PFS was inversely correlated with baseline levels of sVEGFR2 [38]. Alternatively, higher levels of sVEGFR1 and lower levels of sVEGFR2 were related to organ dysfunction in patients with disseminated intravascular coagulation [42]. The role of sVEGFR2 as a biomarker remains is not yet understood, and further analysis will be necessary to examine its potential as a predictive biomarker of lenvatinib activity.

Predictive plasma biomarkers of survival, including PFS and overall survival (OS), may greatly inform patient care and management. Higher baseline VEGF levels in plasma were correlated with shorter OS in sorafenib-treated patients with renal cancer and hepatocellular carcinoma $[43,44]$. Higher baseline levels of VEGF and IL-8 were associated with shorter PFS and OS in sunitinib-treated patients with renal cancer [45]. PFS and OS were not analyzed in this dose-escalation phase I study enrolling patients with various tumor types and treatment history; therefore correlation analyses with survival was not performed. However, our previous report indicated the inverse correlation of lenvatinib treatment duration with baseline levels of $\mathrm{SDF} 1 \alpha$, but not VEGF or IL-8 [17]. Potential predictive biomarkers of PFS and OS for lenvatinib are under investigation in ongoing phase 2 and 3 studies of lenvatinib.

Hypertension and proteinuria are major toxicities of antiangiogenic VEGF inhibitors, and their onset may suggest inhibition of the VEGF/VEGFR pathway. However, the hypothesis that hypertension and proteinuria are biomarkers of response to antiangiogenic drugs remains inconclusive 
[46]. Differences in the definition of toxicity used for correlation analysis and in the study criteria of baseline disease, as well as use of concomitant agents, may affect the analysis. In this study, tumor shrinkage by lenvatinib was significantly correlated with proteinuria, but not with hypertension or fatigue. Because tumor shrinkage by antitumor agents is tumor type-specific, further analysis will be necessary in future phase 2 and 3 studies to examine the predictive value of toxicities for clinical efficacy of lenvatinib.

The PD change in plasma proteins may reflect a biologic response to lenvatinib treatment. In this study, PD biomarker changes were associated with lenvatinib treatment and were diminished during the 1-week rest period. These data suggest that the continuous administration of lenvatinib may maintain clinical activity. This continuous dosing regimen was adopted in subsequent lenvatinib studies $[18,47]$.

\section{Conclusion}

The analysis of lenvatinib-induced changes in the levels of plasma biomarkers related to angiogenesis suggested that angiogenesis inhibition may be correlated with clinical outcomes in patients with a wide range of solid tumors. Further study of the levels of angiogenic PD biomarkers and their potential relation to clinical outcomes with lenvatinib treatment in solid tumor types appears warranted and may inform treatment decisions.

\begin{abstract}
Abbreviations
AEs: Adverse events; AUC: Area under the curve; bFGF: Basic fibroblast growth factor; HGF: Hepatocyte growth factor; IL: Interleukin; OS: Overall survival; PD: Pharmacodynamic; PDGF: Platelet-derived growth factor; PDGFRa: PDGF receptor alpha; PFS: Progression-free survival; PK: Pharmacokinetic; PIGF: Placental growth factor; RECIST: Response Evaluation Criteria in Solid Tumors; SCF: Stem cell factor; SDF1a: Stromal cell-derived factor-1 alpha; TKI: Tyrosine kinase inhibitor; VEGF: Vascular endothelial growth factor; VEGFR: VEGF receptor; sVEGFR: Soluble VEGFR.
\end{abstract}

\section{Competing interests}

NK, KS, YN, and WY are employees of Eisai Co Ltd. All other authors (NY, $Y Y, H N, F K, K N$, and $\Pi$ ) declare that they have no competing interests.

\section{Authors' contributions}

All of the authors have made substantial contributions to the conception and design of this study and the interpretation of data. NY, YY, HN, and TT participated in the acquisition of data in the clinical facility, and KS and YN performed the PK and PD analysis. NK, WY, and TT have been involved in drafting the manuscript and revising it critically for important intellectual content. TT gave final approval for the published version. All authors read and approved the final manuscript.

\section{Acknowledgments}

The authors thank Tadashi Kadowaki and Yasuhiro Funahashi (Biomarkers and Personalized Medicine Core Function Unit, Eisai Co, Ltd) for technical support of PD parameter analyses. This study was funded by Eisai Co, Ltd. We gratefully acknowledge the commitment of participating patients, their families, and the study investigators for their invaluable contribution to this research. We thank Oxford PharmaGenesis, Inc. for editorial services funded by Eisai Co, Ltd.

\section{Author details}

'Oncology Medical Department, Eisai Co, Ltd, Tokyo, Japan. ${ }^{2} J a p a n$ Biostatistics/Biostatistics/Clinical Science, Scientific and Operational Clinical Support Core Function Unit, Eisai Co, Ltd, Tokyo, Japan. ${ }^{3}$ Japan Clinical
Pharmacology/Clinical Pharmacology/Clinical Science, Scientific and Operation Clinical Support Core Function Unit, Eisai Co, Ltd, Tokyo, Japan. ${ }^{4}$ Oncology Clinical Development Section, Japan/Asia Clinical Research Production Creation Unit, Eisai Co, Ltd, Tokyo, Japan. ${ }^{5}$ Department of Thoracic Oncology, National Cancer Center Hospital, Tokyo, Japan. ${ }^{6}$ Department of Gastrointestinal Oncology, National Cancer Center Hospital, Tokyo, Japan. ${ }^{7}$ Shien-Lab and Support Facility of Project Ward, National Cancer Center Hospital, Tokyo, Japan. ${ }^{8}$ Department of Genome Biology, Kinki University School of Medicine, Osaka, Japan.

Received: 19 November 2013 Accepted: 3 July 2014 Published: 21 July 2014

\section{References}

1. Rapisarda A, Melillo G: Overcoming disappointing results with antiangiogenic therapy by targeting hypoxia. Nat Rev Clin Oncol 2012, 9:378-390.

2. Yamamoto N, Tamura T, Yamamoto N, Yamada K, Yamada Y, Nokihara H, Fujiwara Y, Takahashi T, Murakami H, Boku N, Yamazaki K, Puchalski TA, Shin E: Phase I, dose escalation and pharmacokinetic study of cediranib (RECENTIN), a highly potent and selective VEGFR signaling inhibitor, in Japanese patients with advanced solid tumors. Cancer Chemother Pharmacol 2009, 64:1165-1172.

3. Fujisaka $Y$, Yamada $Y$, Yamamoto $N$, Shimizu T, Fujiwara $Y$, Yamada $K$ Tamura T, Watanabe H, Sun YN, Bass MB, Seki M: Phase 1 study of the investigational, oral angiogenesis inhibitor motesanib in Japanese patients with advanced solid tumors. Cancer Chemother Pharmacol 2010, 66:935-943.

4. Asahina $H$, Tamura $Y$, Nokihara $H$, Yamamoto N, Seki $Y$, Shibata T, Goto $Y$, Tanioka M, Yamada Y, Coates A, Chiu YL, Li X, Pradhan R, Ansell PJ, McKeegan EM, McKee MD, Carlson DM, Tamura T: An open-label, phase 1 study evaluating safety, tolerability, and pharmacokinetics of linifanib (ABT-869) in Japanese patients with solid tumors. Cancer Chemother Pharmacol 2012, 69:1477-1486.

5. Ghajar CM, Peinado H, Mori H, Matei IR, Evason K, Brazier H, Almeida D, Koller A, Hajjar KA, Stainier DY, Chen El, Lyden D, Bissell MJ: The perivascular niche regulates breast tumour dormancy. Nat Cell Biol 2013, 15:807-817

6. Loges S, Schmidt T, Carmeliet P: Mechanisms of resistance to anti-angiogenic therapy and development of third-generation anti-angiogenic drug candidates. Genes Cancer 2010, 1:12-25.

7. Ebos JM, Lee CR, Kerbel RS: Tumor and host-mediated pathways of resistance and disease progression in response to antiangiogenic therapy. Clin Cancer Res 2009, 15:5020-5025.

8. Sennino B, McDonald DM: Controlling escape from angiogenesis inhibitors. Nat Rev Cancer 2012, 12:699-709.

9. Shojaei F, Wu X, Malik AK, Zhong C, Baldwin ME, Schanz S, Fuh G, Gerber HP, Ferrara N: Tumor refractoriness to anti-VEGF treatment is mediated by CD11b + Gr1+ myeloid cells. Nat Biotechnol 2007, 25:911-920.

10. Yao JC, Phan A: Overcoming antiangiogenic resistance. Clin Cancer Res 2011, 17:5217-5219.

11. Ebos JM, Lee CR, Christensen JG, Mutsaers AJ, Kerbel RS: Multiple circulating proangiogenic factors induced by sunitinib malate are tumor-independent and correlate with antitumor efficacy. Proc Nat Acad Sci U S A 2007, 104:17069-17074.

12. Mutsaers AJ, Francia G, Man S, Lee CR, Ebos JM, Wu Y, Witte L, Berry S, Moore M, Kerbel RS: Dose-dependent increases in circulating TGF-alpha and other EGFR ligands act as pharmacodynamic markers for optimal biological dosing of cetuximab and are tumor independent. Clin Cancer Res 2009, 15:2397-2405.

13. Matsui J, Funahashi Y, Uenaka T, Watanabe T, Tsuruoka A, Asada M: Multi-kinase inhibitor E7080 suppresses lymph node and lung metastases of human mammary breast tumor MDA-MB-231 via inhibition of vascular endothelial growth factor-receptor (VEGF-R) 2 and VEGF-R3 kinase. Clin Cancer Res 2008, 14:5459-5465.

14. Matsui J, Yamamoto Y, Funahashi Y, Tsuruoka A, Watanabe T, Wakabayashi T, Uenaka T, Asada M: E7080, a novel inhibitor that targets multiple kinases, has potent antitumor activities against stem cell factor producing human small cell lung cancer $\mathrm{H} 146$, based on angiogenesis inhibition. Int J Cancer 2008, 122:664-671. 
15. Ikuta K, Yano S, Trung VT, Hanibuchi M, Goto H, Li Q, Wang W, Yamada T, Ogino H, Kakiuchi S, Uehara H, Sekido Y, Uenaka T, Nishoka Y, Sone S: E7080, a multi-tyrosine kinase inhibitor, suppresses the progression of malignant pleural mesothelioma with different proangiogenic cytokine production profiles. Clin Cancer Res 2009, 15:7229-7239.

16. Boss DS, Glen H, Beijnen JH, Keesen M, Morrison R, Tait B, Copalu W, Mazur A Wanders J, O'Brien JP, Schellens JH, Evans TR: A phase I study of E7080, a multitargeted tyrosine kinase inhibitor, in patients with advanced solid tumours. Br J Cancer 2012, 106:1598-1604.

17. Yamada K, Yamamoto N, Yamada Y, Nokihara H, Fujiwara $Y$, Hirata T, Koizumi F, Nishio K, Koyama N, Tamura T: Phase I dose-escalation study and biomarker analysis of E7080 in patients with advanced solid tumors. Clin Cancer Res 2011, 17:2528-2537.

18. Sherman SI, Jarzab B, Cabanillas ME, Licitra LF, Pacini F, Martins R, Robinson B, Ball D, McCaffrey J, Shah MH, Bodenner D, Allison R, Newbold K, Elisei R, O'Brien JP, Schlumberger M: A phase II trial of the multitargeted kinase inhibitor E7080 in advanced radioiodine (RAl)-refractory differentiated thyroid cancer (DTC) [abstract]. J Clin Oncol 2011, 29(suppl):5503.

19. Keizer RJ, Gupta A, Shumaker R, Beijnen JH, Schellens JH, Huitema AD: Model-based treatment optimization of a novel VEGFR inhibitor. $\mathrm{Br} J \mathrm{Clin}$ Pharmacol 2012, 74:315-326.

20. Therasse P, Arbuck SG, Eisenhauer EA, Wanders J, Kaplan RS, Rubinstein L, Verweij J, Van Glabbeke M, van Oosterom AT, Christian MD, Gwyther SG: New guidelines to evaluate the response to treatment in solid tumors. European Organization for Research and Treatment of Cancer, National Cancer Institute of the United States, National Cancer Institute of Canada. J Natl Cancer Inst 2000, 92:205-216.

21. Zhu X, Wu S, Dahut WL, Parikh CR: Risks of proteinuria and hypertension with bevacizumab, an antibody against vascular endothelial growth factor: systematic review and meta-analysis. Am J Kidney Dis 2007 49:186-193.

22. Robinson ES, Matulonis UA, Ivy P, Berlin ST, Tyburski K, Penson RT, Humphreys BD: Rapid development of hypertension and proteinuria with cediranib, an oral vascular endothelial growth factor receptor inhibitor. Clin J Am Soc Nephrol 2010, 5:477-483.

23. Ferrara $\mathrm{N}$ : Vascular endothelial growth factor and the regulation of angiogenesis. Recent Prog Horm Res 2000, 55:15-35.

24. Makino H, Aoki M, Hashiya N, Yamasaki K, Azuma J, Sawa Y, Kaneda Y, Ogihara T, Morishita R: Long-term follow-up evaluation of results from clinical trial using hepatocyte growth factor gene to treat severe peripheral arterial disease. Arterioscler Thromb Vasc Biol 2012, 32:2503-2509.

25. Guo Y, Su L, Li Y, Guo N, Xie L, Zhang D, Zhang X, Li H, Zhang G, Wang Y, Liu C: The synergistic therapeutic effect of hepatocyte growth factor and granulocyte colony-stimulating factor on pulmonary hypertension in rats. Heart Vessels 2013. Aug 10: Epub ahead of print

26. Mills PJ, Parker B, Dimsdale JE, Sadler GR, Ancoli-Israel S: The relationship between fatigue and quality of life and inflammation during anthracycline-based chemotherapy in breast cancer. Biol Psychol 2005, 69:85-96.

27. Kanehisa M, Ishitobi $Y$, Ando T, Okamoto S, Maruyama Y, Kohno K, Ninomiya T, Higuma H, Tanaka Y, Tsuru J, Hanada H, Kodama K, Akiyoshi J: Serum hepatocyte growth factor levels and the effects of antidepressants in panic disorder. Neuropeptides 2010, 44:431-435

28. Ravaud A, Schmidinger M: Clinical biomarkers of response in advanced renal cell carcinoma. Ann Oncol 2013, 24:2935-2942.

29. Rutkowski P, Bylina E, Klimczak A, Switaj T, Falkowski S, Kroc J, Lugowska I, Brzeskwiniewicz M, Melerowicz W, Osuch C, Mierzejewska E, Wasielewski K Woźniak A, Grzesiakowska U, Nowecki Zl, Siedlecki JA, Limon J: The outcome and predictive factors of sunitinib therapy in advanced gastrointestinal stromal tumors (GIST) after imatinib failure - one institution study. BMC Cancer 2012, 12:107

30. Yang Y, Zhang Y, Cao Z, Ji H, Yang X, Iwamoto H, Wahlberg E, Länne T, Sun B, Cao Y: Anti-VEGF- and anti-VEGF receptor-induced vascular alteration in mouse healthy tissues. Proc Natl Acad Sci U S A 2013, 110:12018-12023.

31. Fujiwara $Y$, Kiyota N, Chayahara N, Suzuki A, Umeyama Y, Mukohara T, Minami H: Management of axitinib (AG-013736)-induced fatigue and thyroid dysfunction, and predictive biomarkers of axitinib exposure: results from phase I studies in Japanese patients. Invest New Drugs 2012, 30:1055-1064.

32. Noda M, Omatsu Y, Sugiyama T, Oishi S, Fujii N, Nagasawa T: CXCL12-CXCR4 chemokine signaling is essential for NK-cell development in adult mice. Blood 2011, 117:451-458.
33. Williams SA, Harata-Lee $Y$, Comerford I, Anderson RL, Smyth MJ, McColl SR: Multiple functions of CXCL12 in a syngeneic model of breast cancer. Mol Cancer 2010, 9:250.

34. Jain RK, Duda DG, Willett CG, Sahani DV, Zhu AX, Loeffler JS, Batchelor TT, Sorensen AG: Biomarkers of response and resistance to antiangiogenic therapy. Nat Rev Clin Oncol 2009, 6:327-338.

35. Ferrara N: Vascular endothelial growth factor as a target for anticancer therapy. Oncologist 2004, 9(Suppl 1):2-10.

36. Thomas CP, Andrews JI, Liu KZ: Intronic polyadenylation signal sequences and alternate splicing generate human soluble Flt1 variants and regulate the abundance of soluble Flt1 in the placenta. FASEB J 2007, 21:3885-3895.

37. Albuquerque RJ, Hayashi T, Cho WG, Kleinman ME, Dridi S, Takeda A, Baffi JZ, Yamada K, Kaneko H, Green MG, Chappell J, Wilting J, Weich HA, Yamagami S, Amano S, Mizuki N, Alexander JS, Peterson ML, Brekken RA, Hirashima M, Capoor S, Usui T, Ambati BK, Ambati J: Alternatively spliced vascular endothelial growth factor receptor-2 is an essential endogenous inhibitor of lymphatic vessel growth. Nat Med 2009, 15:1023-1030.

38. Zhu AX, Ancukiewicz M, Supko JG, Sahani DV, Blaszkowsky LS, Meyerhardt JA Abrams TA, McCleary NJ, Bhargava P, Muzikansky A, Sheehan S, Regan E, Vasudev E, Knowles M, Fuchs CS, Ryan DP, Jain RK, Duda DG: Efficacy, safety, pharmacokinetics, and biomarkers of cediranib monotherapy in advanced hepatocellular carcinoma: a phase II study. Clin Cancer Res 2013, 19:1557-1566.

39. Batchelor TT, Sorensen AG, di Tomaso E, Zhang WT, Duda DG, Cohen KS, Kozak KR, Cahill DP, Chen PJ, Zhu M, Ancukiewicz M, Mrugala MM, Plotkin S, Drappatz J, Louis DN, Ivy P, Scadden DT, Benner T, Loeffler JS, Wen PY, Jain RK: AZD2171, a pan-VEGF receptor tyrosine kinase inhibitor, normalizes tumor vasculature and alleviates edema in glioblastoma patients. Cancer Cell 2007, 11:83-95.

40. Motzer RJ, Michaelson MD, Redman BG, Hudes GR, Wilding G, Figlin RA, Ginsberg MS, Kim ST, Baum CM, DePrimo SE, Li JZ, Bello CL, Theuer CP, George DJ, Rini BI: Activity of SU11248, a multitargeted inhibitor of vascular endothelial growth factor receptor and platelet-derived growth factor receptor, in patients with metastatic renal cell carcinoma. J Clin Oncol 2006, 24:16-24.

41. Tomita $Y$, Uemura H, Fujimoto H, Kanayama HO, Shinohara N, Nakazawa $H$, Imai K, Umeyama Y, Ozono S, Naito S, Akaza H: Key predictive factors of axitinib (AG-013736)-induced proteinuria and efficacy: a phase II study in Japanese patients with cytokine-refractory metastatic renal cell carcinoma. Eur J Cancer 2011, 47:2592-2602.

42. Jesmin S, Wada T, Gando S, Sultana SS, Zaedi S: The dynamics of angiogenic factors and their soluble receptors in relation to organ dysfunction in disseminated intravascular coagulation associated with sepsis. Inflammation 2013, 36:186-196.

43. Escudier B, Eisen T, Stadler WM, Szczylik C, Oudard S, Staehler M, Negrier S, Chevreau C, Desai AA, Rolland F, Demkow T, Hutson TE, Gore M, Anderson S, Hofilena G, Shan M, Pena C, Lathia C, Bukowski RM: Sorafenib for treatment of renal cell carcinoma: final efficacy and safety results of the phase III treatment approaches in renal cancer global evaluation trial. J Clin Oncol 2009, 27:3312-3318.

44. Llovet JM, Peña CE, Lathia CD, Shan M, Meinhardt G, Bruix J: Plasma biomarkers as predictors of outcome in patients with advanced hepatocellular carcinoma. Clin Cancer Res 2012, 18:2290-2300.

45. Harmon CS, DePrimo SE, Figlin RA, Hudes GR, Hutson TE, Michaelson MD, Négrier S, Kim ST, Huang X, Williams JA, Eisen T, Motzer RJ: Circulating proteins as potential biomarkers of sunitinib and interferon-a efficacy in treatment-naïve patients with metastatic renal cell carcinoma. Cancer Chemother Pharmacol 2014, 73:151-161.

46. Horsley L, Marti K, Jayson GC: Is the toxicity of anti-angiogenic drugs predictive of outcome? A review of hypertension and proteinuria as biomarkers of response to anti-angiogenic therapy. Expert Opin Drug Metab Toxicol 2012, 8:283-293.

47. Nishio M, Horai T, Horiike A, Nokihara H, Yamamoto N, Takahashi T, Murakami H, Yamamoto N, Koizumi F, Nishio K, Yusa W, Koyama N, Tamura T: Phase 1 study of lenvatinib combined with carboplatin and paclitaxel in patients with non-small-cell lung cancer. Br J Cancer 2013, 109:538-544.

doi:10.1186/1471-2407-14-530

Cite this article as: Koyama et al:: Pharmacodynamic change in plasma angiogenic proteins: a dose-escalation phase 1 study of the multi-kinase inhibitor lenvatinib. BMC Cancer 2014 14:530. 